\title{
Evaluación de un proceso de bioestimulación aplicado a suelos áridos contaminados con hidrocarburos del petróleo.
}

\section{Assessment of a biostimulation process applied to petroleum hydrocarbon-contaminated arid soils}

Presentación: 6-7/10/2020

\section{Doctorando:}

\section{Andrea Pojmaevich}

Ingeniería Química Aplicada a los Bioprocesos (IQAB) - Facultad Regional del Neuquén - Universidad Tecnológica Nacional - Argentina

abpojmaevich@gmail.com

\section{Director/es:}

Víctor Busto

\section{Co-director/es:}

\section{Alberto G. Camacho}

\section{Resumen}

La biorremediación ha demostrado ser una técnica eficaz para la recuperación de suelos contaminados con hidrocarburos, especialmente porque no genera otros residuos que requieran ser tratados, además de ser un proceso económicamente viable y amigable con el medio ambiente. Es sabido que el mejoramiento de las condiciones ambientales y nutricionales de la microflora autóctona de los suelos puede incrementar significativamente los procesos de biodegradación lo cual puede lograrse modificando la humedad y la disponibilidad de nutrientes ( $\mathrm{N}, \mathrm{P}$ y $\mathrm{O}_{2}$ fundamentalmente) en suelos pobres, es decir, aplicando una técnica de bioestimulación. El objetivo de este trabajo fue evaluar la capacidad biodegradadora de los microorganismos autóctonos presentes en un suelo de tipo árido contaminado con hidrocarburos del petróleo (HTP) de la zona de Catriel Oeste (cuenca neuquina), mediante una técnica de bioestimulación en microcosmos.

Se utilizaron sistemas microcosmos conteniendo $200 \mathrm{~g}$ de suelo, los cuales fueron bioestimulados (BIO) ajustando la proporción de nutrientes (C:N:P 100:10:1). Por otra parte, se realizaron controles comunidad (CC) en microcosmos sin bioestimular. Ambos sistemas se incubaron a $25^{\circ} \mathrm{C}$ manteniendo la humedad al $15 \%$ y se mezclaron periódicamente, cada 3 días, con el objeto de airear el suelo. Se tomaron muestras al inicio, a los 28 y a los 42 días, y se determinaron la concentración de HTP, actividad microbiana total (AMT), recuento de bacterias heterótrofas aerobias totales (BHAT) y bacterias degradadoras totales (BDT).

Ambos sistemas evaluados (CC y BIO) mostraron una reducción significativa del contenido de HTP (88\%), la cual estaría relacionada con los incrementos observados en la AMT (4,3 y 3,7 veces para los sistemas CC y BIO, respectivamente), y con el aumento del número de BHAT y BDT (2 órdenes de magnitud en ambos sistemas).

Se puede concluir que la aireación del suelo parece ser un mecanismos simple y adecuado para la remoción de hidrocarburos en este suelo y que la aplicación de una técnica de bioestimulación no mejora la remoción del sistema donde solo se contempló la incorporación de aire a través del mezclado. Esto sugiere que es conveniente el desarrollo de un proceso que mejore las condiciones de aireación con el objeto de favorecer en mayor medida la biodegradación de los hidrocarburos del petróleo. 


\begin{abstract}
Bioremediation proved to be an effective approach to deal with hydrocarbon-contaminated soils, mainly because it does not generate wastes that need to be treated, in addition to being an economically viable process. It is well known that improvement of the growth conditions of soil native microbiota can significantly increase bioremediation processes. The latter can be achieved by modifying the humidity and the availability of nutrients in poor soils using a biostimulation approach. The aim of the present work was to evaluate the biodegrading capacity of the native microbiota present in a petroleum hydrocarbon-contaminated arid soil (HTP) from Catriel Oeste area (Neuquén basin), using a biostimulation technique.

Microcosm systems containing $200 \mathrm{~g}$ of soil were biostimulated (BIO) by adjusting the proportion of nutrients (C: N: P 100: 10: 1). Moreover, community controls (CC) were carried out in microcosm without biostimulation. Both systems were incubated at $25^{\circ} \mathrm{C}$ keeping the humidity at $15 \%$ and mixed in order to aerate the soil. Samples were withdrawn at 0,28 and 42 days. HTP concentration, total microbial activity (AMT), total aerobic bacteria (BHAT) and total degrading bacteria (BDT) were determined.

In both systems (CC and BIO) a significant decrease in the HTP content (88\%) was achieved. This phenomenon would be related to AMT increases (4.3 and 3.7 fold increases for CC and BIO systems, respectively), and to BHAT and BDT increases (two orders of magnitude higher in both systems).

It can be concluded that a biostimulation technique would not be effective for these soils compared to the incorporation of air through mixing. These results would lead to a process development in order to improve aeration conditions aimed to increase petroleum hydrocarbons biodegradation.
\end{abstract}

Keywords: biorremediation, biostimulation, contaminated soils, petroleum hydrocarbons

\title{
Introducción
}

En la cuenca neuquina se ha incrementado en las últimas décadas la producción hidrocarburífera, convirtiéndose en uno de los pilares fundamentales de la economía regional asociados principalmente al cobro de regalías, la generación de empleo y actividades comerciales referidas a las etapas del proceso de exploración y explotación (Landriscini et al., 2017). Así como ha aumentado la producción, han aumentado los pasivos ambientales generados por esta industria y han provocado históricamente la contaminación de suelos, napas y cursos de agua superficiales, situación que, en estos tiempos aún continúa, sea por falta o fallas en el mantenimiento, por descuidos, inclemencias climáticas o vandalismo. Si bien, tradicionalmente la eliminación de este tipo de contaminantes se ha realizado utilizando metodologías que involucran tratamientos físicos o químicos, (Araruna et al., 2004), éstos no han sido suficientes ya que no se han reparado en su mayoría los sitios con contaminación histórica, así es que, hace ya algunos años se han sumado los procesos biotecnológicos, que sustituyen o complementan los sistemas tradicionales. Los procesos biotecnológicos utilizados en general implican el retiro del material de la zona de incumbencia para tratarlos en repositorios donde se mezclan con suelos contaminados de otros sectores y con otros hidrocarburos de forma ex-situ (Benavides López de Mesa et al., 2006).

Dentro de estos tratamientos la biorremediación ha demostrado ser una de las técnicas más eficientes de remediación de suelos contaminados con hidrocarburos, la cual puede ser definida como la utilización de organismos vivos para reducir o eliminar riesgos medioambientales resultantes de la acumulación de compuestos químicos tóxicos y otros residuos peligrosos (González Rojas, 2011).

Los factores que gobiernan la biorremediación son, factores del hidrocarburo como la composición química, el estado físico y la concentración; factores ambientales como la temperatura, disponibilidad de oxígeno, humedad, salinidad, nutrientes, $\mathrm{pH}$, y factores microbianos como la cantidad, dotación enzimática, asociación por co-oxidación, dinámica de las poblaciones y velocidad de biodegradación (Luis et al., 2020; Salomón, 2020). Claro que naturalmente el ambiente tiene cierta capacidad de amortiguar o subsanar los efectos de los contaminantes, dependiendo claro de qué tipo sean, de su concentración y frecuencia de ocurrencia, este puede llevar mucho tiempo antes de conseguir las condiciones originales del ambiente en cuestión, tal como sucede con los hidrocarburos del petróleo. Teniendo en cuenta estas consideraciones, es sabido que el mejoramiento de las condiciones de crecimiento de la microflora autóctona de los suelos puede incrementar significativamente los procesos de remediación. Esto puede lograrse modificando la humedad y la disponibilidad de nutrientes en suelos pobres, es decir, aplicando un proceso de bioestimulación (Martínez Álvarez et al., 2017).

Estudios previos realizados por nuestro grupo de investigación demostraron que suelos contaminados con hidrocarburos procedentes de la localidad de Catriel (cuenca neuquina) poseen una microflora con capacidad 
biodegradadora de los contaminantes presentes, lo que los hacen susceptibles de ser tratados mediante una adecuada estrategia de biorremediación (Pojmaevich et al., 2019). Además, se demostró que estos suelos carecen de nutrientes tales como nitrógeno y fósforo, ya que ambos arrojaron valores inferiores al límite de detección de las técnicas analíticas.

Teniendo en cuenta estos antecedentes, el objetivo de este trabajo fue evaluar la capacidad biodegradadora de los microorganismos autóctonos presentes en un suelo contaminado con hidrocarburos del petróleo (HTP) de la cuenca neuquina mediante una estrategia de bioestimulación en microcosmos.

\section{Desarrollo}

Para el desarrollo del presente trabajo se tomaron muestras de suelo contaminado con HTP de la localidad de Catriel (cuenca neuquina) por gentileza de la empresa Aconcagua Energía S.A, quien está a cargo de la operación de la Base operativa Catriel Oeste, concesionada a la empresa Provincial de Rio Negro, EDHIPSA. Este suelo sufrió un derrame de petróleo crudo seis meses antes del muestreo, el cual fue llevado a cabo tomando en consideración la guía para muestreo de suelos que sigue los Estándares de Calidad Ambiental para Suelo.

Las muestras de suelo fueron acondicionadas realizando una limpieza manual para retirar porciones de mayor tamaño, y luego fueron tamizadas mediante una malla de $1 \mathrm{~mm}$.

Inicialmente se realizó la caracterización de los suelos determinando el $\mathrm{pH}$, el \% de humedad utilizando el método gravimétrico descripto por Fernandez Linares et al., (2006), y el contenido de hidrocarburos totales del petróleo (HTP) (EPA 418.1, 1978; Villacreses, 2011) expresado en mg HTP/Kg de suelo seco (SS).

Se diseñaron cuatro sistemas microcosmos utilizando frascos de boca ancha $(10 \mathrm{~cm}$ de alto y $5 \mathrm{~cm}$ de diámetro) conteniendo 200 g de suelo contaminado cada uno, de acuerdo a lo descripto por Ruberto et al. (2003). Estos microcosmos fueron divididos en dos grupos: dos de ellos (duplicado) fueron bioestimulados (BIO) adicionando una solución de sales conteniendo 1,6231 g de $\mathrm{NH}_{4} \mathrm{Cl}$ y 0,1947 g de $\mathrm{Na}_{2} \mathrm{HPO}_{4}$, con el objeto de ajustar la proporción de nutrientes (C:N:P) en una relación de 100:10:1, según sugiere Martínez Álvarez et al. (2015). Para estimar las cantidades de sales de nitrógeno y fósforo a adicionar se tuvo en cuenta la concentración inicial de HTP del suelo contaminado, el cual resultó para nuestro caso de 27,78 g HTP/Kg de SS. Por otro lado, otros dos microcosmos (duplicado) fueron utilizados como control comunidad (CC), sin bioestimular. Todos los sistemas microcosmos se incubaron a $25^{\circ} \mathrm{C}$ durante 42 días ajustando periódicamente la humedad al $15 \%$, ya que se trabajó con un suelo de zona árida, y se mezclaron (cada 3 días) con el objeto de airear el suelo.

La evolución de los procesos de bioestimulación y biorremediación se siguieron tomando muestras de suelo al inicio, a los 28 y a los 42 días, a las que se les determinaron la concentración de HTP, el número de bacterias heterótrofas aerobias totales (BHAT) y bacterias degradadoras totales (BDT) (Hanson et al., 1993), y la actividad microbiana total (AMT) (Adam \& Duncan, 2001), medida como $\mu \mathrm{g}$ de fluoresceína por gramo de suelo seco ( $\mu \mathrm{g}$ fluoresceína/g SS).

\section{Resultados}

La Figura 1 muestra la evolución del contenido de HTP en función del tiempo en los sistemas BIO y CC. Allí se observó una marcada disminución del contenido de HTP partiendo de un valor inicial de $26443 \mathrm{mg} . \mathrm{kg}^{-1} \mathrm{y}$ alcanzando un valor de 5538 mg.kg-1 para los sistemas CC y de 5285 mg.kg-1 para los sistemas BIO al cabo de 28 días, lo que indica un porcentaje de remoción de HTP del 79\% en promedio. Sin embargo, este porcentaje de degradación se incrementó aún más alcanzando un valor de $88 \%$ en promedio al cabo de 42 días.

Por otra parte, no se observaron diferencias significativas entre ambos sistemas evaluados (CC y BIO). Estos resultados podrían indicar que la microflora presente en este suelo estaría adaptada a la presencia de los HTP producto de la contaminación original y a las condiciones de aridez del suelo, ya que la incorporación de los nutrientes en los sistemas BIO no incrementaron el porcentaje de remoción en relación a los sistemas CC. Por lo tanto, la remoción aquí observada podría deberse fundamentalmente al mantenimiento de los porcentajes de humedad del suelo y a la incorporación de aire en el proceso de mezclado. 


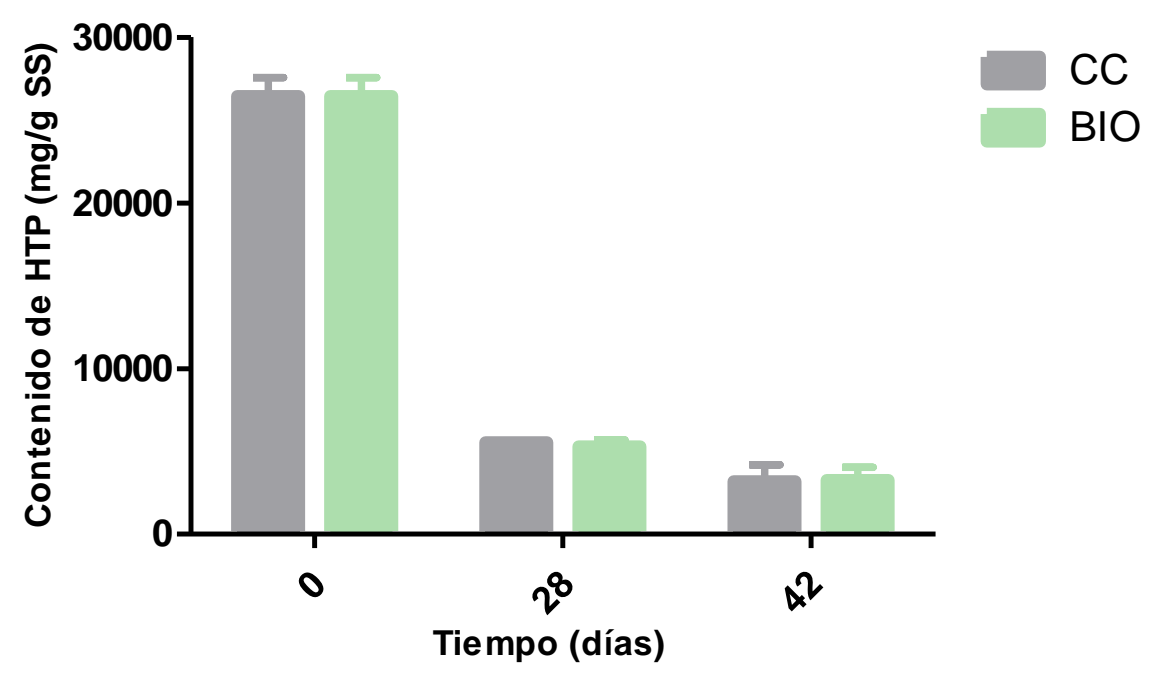

Fig. 1: Concentración de hidrocarburos totales del petróleo (HTP) en función del tiempo en los sistemas control comunidad (CC) y bioestimulados (BIO).

En cuanto a la actividad biológica total (AMT) mostrada en la Figura 2, se observó un aumento gradual de la misma desde un valor inicial de 1,22 $\mu$ g fluoresceína.g-1 ${ }^{-1}$ S hasta un valor de 5,24 $\mu$ g fluoresceína.g- ${ }^{-1}$ SS para los sistemas CC, y de 4,65 $\mu \mathrm{g}$ fluoresceína.g- ${ }^{-1}$ SS para los sistemas BIO, al cabo de 42 días. Estos incrementos fueron de 4,3 y 3,7 veces para los sistemas CC y BIO, respectivamente.

Los valores alcanzados al cabo de los 42 días no mostraron diferencias significativas, lo que al igual que en el contenido de HTP, se podría inferir que la adición de nutrientes no conduciría a un incremento significativo de la AMT en los sistemas BIO respecto de los sistemas CC.

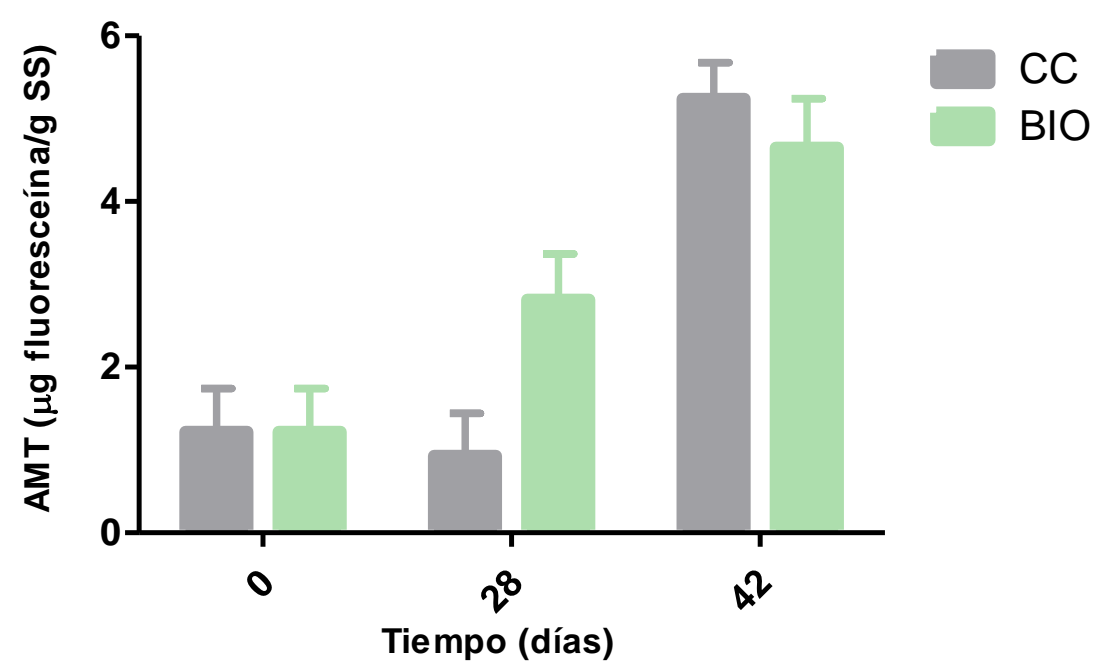

Fig. 2: Actividad biológica total del suelo (AMT), evaluada como fluoresceína liberada,en función del tiempo en los sistemas control comunidad (CC) y bioestimulados (BIO).

En relación al recuento de bacterias presentes en los sistemas CC y BIO, las aerobias totales (BHAT) fueron cultivadas en medio R2A y las degradadoras totales de hidrocarburos (BDT) en agar gasoil (MSB + gasoil). Ambos tipos de bacterias mostraron un aumento de las UFC/g SS en función del tiempo. Para el caso de las BHAT (Figura 3),

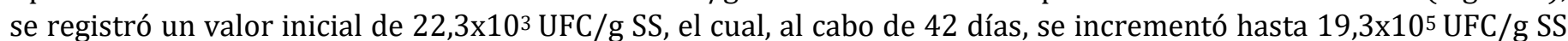
en los sistemas CC y hasta $66 \times 10^{5} \mathrm{UFC} / \mathrm{g}$ SS en los sistemas BIO, lo que indicaría un incremento de dos órdenes de magnitud para ambos sistemas. 
Por otra parte, en la Figura 4, se observa un incremento de las BDT el cual resultó de dos órdenes de magnitud,

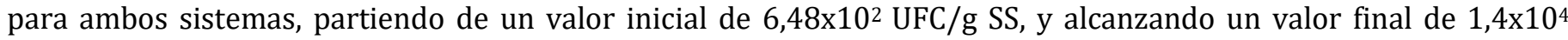
UFC/g SS y de 1,9 x104 UFC/g SS para los sistemas CC y BIO, respectivamente.

Tanto para BHAT como para BDT, los valores alcanzados al cabo de 42 días no mostraron diferencias significativas entre los sistemas CC y BIO, y los incrementos registrados en UFC/g SS fueron similares (2 órdenes de magnitud).

Estos incrementos observados en el número de bacterias (BHAT y BDT), estarían en relación directa con los aumentos de la AMT, lo que explicaría el elevado porcentaje de remoción de HTP (88\%) observado en los sistemas CC y BIO. Además, la ausencia de diferencias significativas observada entre ambos tipos de sistemas, manifestaría en mayor medida la importancia de la incorporación de aire en los procesos de remoción de HTP, en relación a la bioestimulación de la microflora autóctona por incorporación de nutrientes en este tipo de suelos.

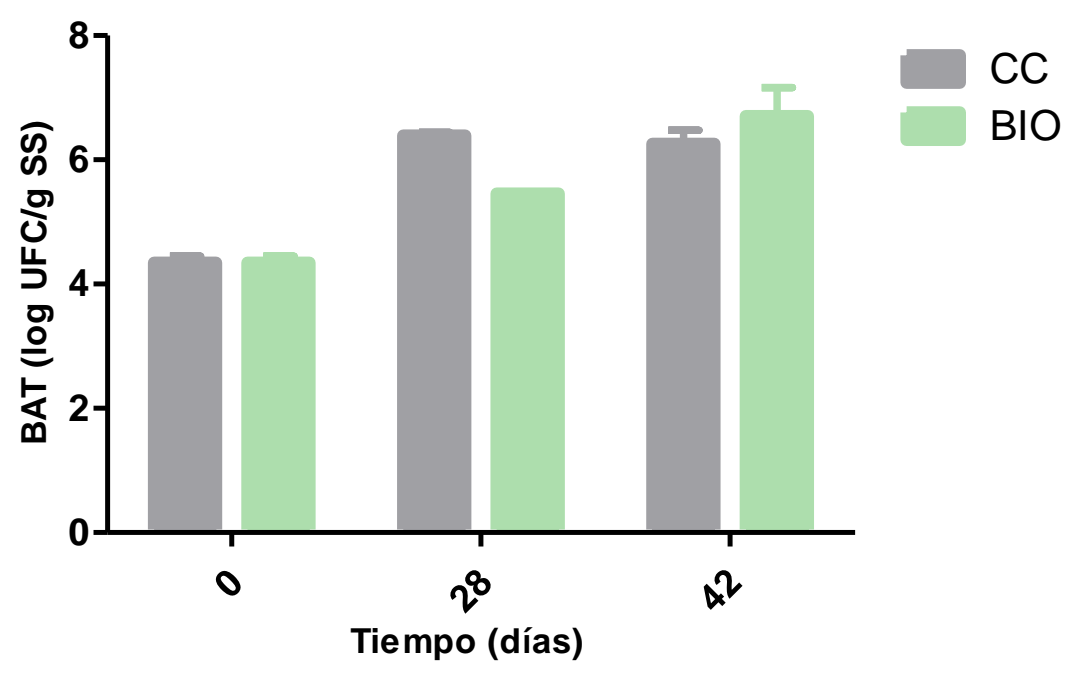

Fig. 3: Recuento de bacterias aerobias totales (BHAT), expresado en escala logarítmica de UFC/g SS, en función del tiempo en los sistemas control comunidad (CC) y bioestimulados (BIO).

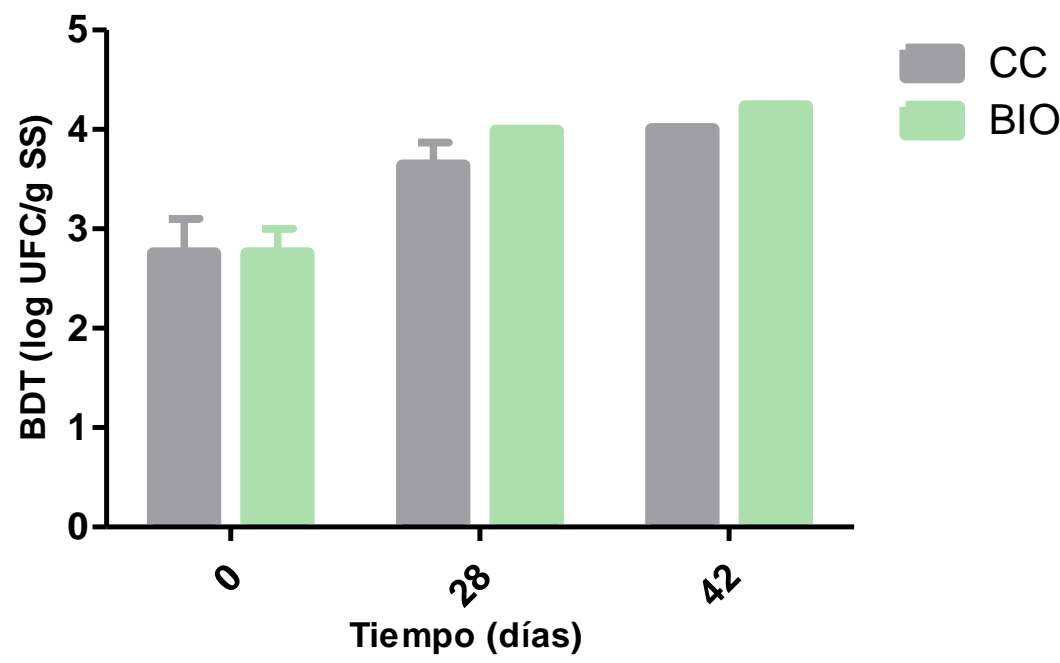

Fig. 4: Recuento de bacterias degradadoras totales (BDT), expresado en escala logarítmica de UFC/g SS, en función del tiempo en los sistemas control comunidad (CC) y bioestimulados (BIO). 


\section{Conclusiones}

En ambos sistemas evaluados (CC y BIO) se alcanzó una reducción significativa del contenido de HTP (88\%), la cual se mostró acompañada por incrementos en la AMT y en el número de BHAT y BDT.

Por lo tanto, se puede concluir que la aplicación de una técnica de bioestimulación no aportaría una ventaja para este tipo de suelos, con el nivel de contaminación y el aparente grado de adaptación de la microflora autóctona, en comparación con la incorporación de aire mediante un proceso de mezclado. Estos resultados permitirían el posible desarrollo de un proceso que mejore las condiciones de aireación en este tipo de suelos, con el objeto de favorecer en mayor medida la biodegradación de los hidrocarburos del petróleo.

\section{Referencias}

Adam, G., \& Duncan, H. (2001). Development of a sensitive and rapid method for the measurement of total microbial activity using fluorescein diacetate (FDA) in a range of soils. Soil Biology and Biochemistry, 33(7-8), 943-951. https://doi.org/10.1016/S0038-0717(00)00244-3

Araruna, J. T., Portes, V. L. O., Soares, A. P. L., Silva, M. G., Sthel, M. S., Schramm, D. U., Tibana, S., \& Vargas, H. (2004). Oil spills debris clean up by thermal desorption. Journal of Hazardous Materials, 110(1-3), 161-171. https://doi.org/10.1016/j.jhazmat.2004.02.054

Benavides López de Mesa; MSc, J., Quintero, MSc, G., Guevara Vizcaíno, A. L., Jaimes Cáceres, D. C., Gutiérrez Riaño, S. M., \& Miranda García, J. (2006). Bioremediación de suelos contaminados con hidrocarburos derivados del petróleo. Nova, 4(5), 82. https://doi.org/10.22490/24629448.351

Del, U., En, S., \& Prístino, B. (2014). Hidrólisis de fluoresceína diacetato y actividad de las enzimas proteasa, celulasa, nitrato reductasa y ureasa del suelo en bosque prístino, sur de chile.

EPA 418.1. (1978). Method 418.1: petroleum hydrocarbons (spectrophotometric, infrared). Issued, 3.

Fernandez Linares, L. carlos, Rojas Avelizapa, N. G., Roldán Castillo, T. G., Ramírez Islas, M. E., Zegarra Martínez, H. G., Uribe Hernández, R., Reyes Ávila, R. J., Flores Hernández, D., \& Arce Ortega, J. M. (2006). Manual de técnicas de análisis de suelos aplicadas a la remediación de sitios contaminados (Issue 1). https://doi.org/10.16309/j.cnki.issn.10071776.2003.03.004

Fontvieille, D. A., Outaguerouine, A., \& Thevenot, D. (2015). Measure of Microbial Activity in Aquatic Systems : Application To Activated Sludges.

González Rojas, E. H. (2011). Concepto y estrategias de biorremediación. Inge@uan, 1(1), 20-29.

Hanson, K. G., Desai, J. D., \& Desai, A. J. (1993). A rapid and simple screening technique for potential crude oil degrading microorganisms. Biotechnology Techniques, 7(10), 745-748. https://doi.org/10.1007/BF00152624

Landriscini, G. S., Preiss, O., \& Avellá, B. (2017). El desarrollo reciente de los hidrocarburos en la Cuenca Neuquina: cambios funcionales en el sistema urbano regional y localización industrial. 48, 1-16. http://mundourbano.unq.edu.ar/index.php/ultimo-numero/276-el-desarrollo-reciente-de-los-hidrocarburos-enla-cuenca-neuquina-cambios-funcionales-en-el-sistema-urbano-regional-y-localizacion-industrial

Luis, R. J., Cruz, I., Luis, J., Guadalupe, M. P., Manuel, S. J., \& Artículo, D. (2020). Recuperación de un suelo contaminado por una mezcla de hidrocarburos Recovering of soil polluted by hydrocarbons mixing Resumen. July.

Martínez Álvarez, L. M., Lo Balbo, A., Mac Cormack, W. P., \& Ruberto, L. A. M. (2015). Bioremediation of a petroleum hydrocarbon-contaminated Antarctic soil: Optimization of a biostimulation strategy using response-surface methodology (RSM). Cold Regions Science and Technology, 119, 61-67. https://doi.org/10.1016/j.coldregions.2015.07.005

Martínez Álvarez, L. M., Ruberto, L. A. M., Lo Balbo, A., \& Mac Cormack, W. P. (2017). Bioremediation of hydrocarboncontaminated soils in cold regions: Development of a pre-optimized biostimulation biopile-scale field assay in Antarctica. Science of the Total Environment, 590-591, 194-203. https://doi.org/10.1016/j.scitotenv.2017.02.204

Pojmaevich, A., Demaría, I., Cruz, M., Pincheira, J., Camacho, A., Ruberto, L., \& Busto, V. (2019). ESTUDIO DE Biodegradabilidad in vitro de hidrocarburos del petróleo en suelos contaminados de Catriel Oeste (Cuenca Neuquina). Editorial Facultad de Agronomía.

Salomón, J. S. C. (2020). BIORREMEDIACIÓN DE HIDROCARBUROS SATURADOS Y AROMÁTICOS POLICÍCLICOS Chiriví. In $\begin{array}{llllll}\text { Libros } & \text { Universidad } & \text { Abierta } & \text { y } & \text { a }\end{array}$ https://hemeroteca.unad.edu.co/index.php/book/article/view/3991

Villacreses, L. (2011). Evaluación de factores que afectan la medición de hidrocarburos totales de petróleo (HTP) mediante espectroscopia IR en suelos contaminados con petróleo intemperizado. (p. 7). 